\title{
An unusual complication following replacement of the aortic valve by the Starr-Edwards prosthesis
}

\author{
B. BEDNA ̌́ ÍK, K. HOF M A N N, O. MED Ř I C K Ý, \\ J. NAVRÁTIL, ANDO. OLEJNÍK \\ From the Second Surgical Clinic, Brno, Czechoslovakia
}

At the 2nd Surgical Clinic of Brno we replace the aortic valve by a Starr-Edwards prosthesis in suitable patients. The technique we use is as follows :

The prosthesis is sutured to the aortic wall by single 4-0 orsilon stitches after the deformed cusps of the valve have been excised. We place the stitches in an axial direction, parallel to one another, and in one row. They are passed below the orifices of the coronary arteries and pass through the original attachment of the aortic valve. We also pass one stitch into each of the three commissures; the other stitches, being three to four in number according to the size of the valve, we pass into the remaining valve cusp. After this we pass these stitches through the teflon cuff of the prosthesis. When all the stitches are in place we put the prosthesis into the aorta under the orifice of the coronary arteries and tie the stitches.

The object of this communication is to describe an unusual complication that occurred during the replacement of an aortic valve in the way described above. There were no unusual features about the clinical condition or the operative technique in the patient concerned. He was a man aged 45 suffering from calcific aortic stenosis. The artificial heart-lung apparatus was used and the valve was sutured into position during extracorporeal circulation. When the operation on the valve had been completed there was some difficulty in resuscitating the heart. The beat was weak and episodes of ventricular fibrillation occurred. It was necessary on several occasions to resume extracorporeal circulation, and eventually pulmonary oedema occurred which was rapidly followed by death. Necropsy revealed that the valve had been sutured into position correctly (Fig. 1). The orifices of both coronary arteries were free and all the stitches had been tied accurately and without a dehiscence. When the left ventricle was opened, however, it was

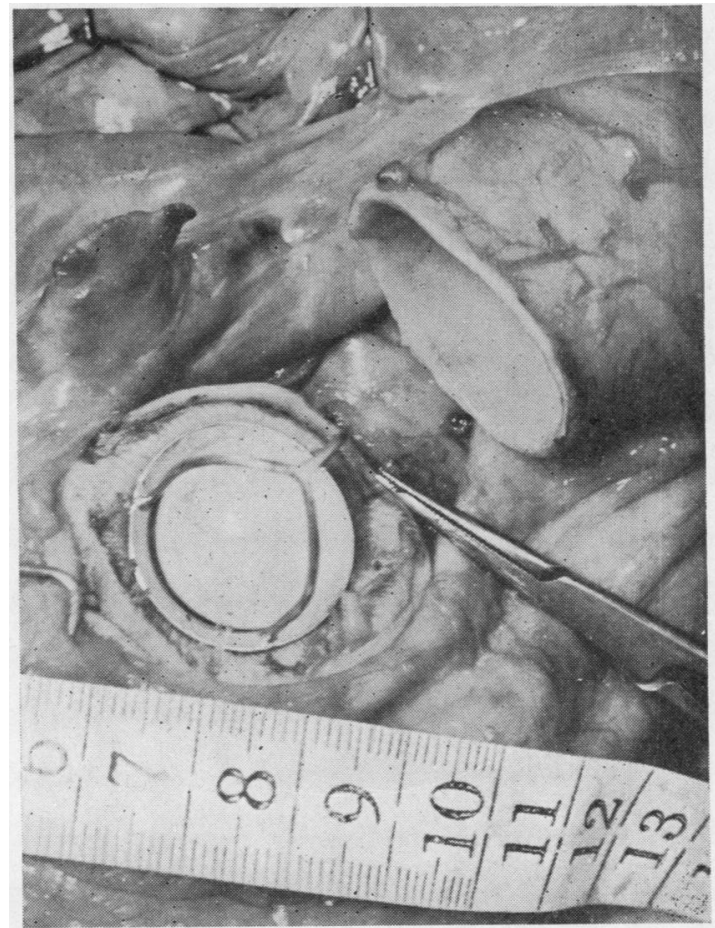

FIG. 1. Post-mortem findings. View of the implanted surface from the aortal side.

immediately apparent that loops from two of the stitches had passed across the base of the valve, thus preventing the ball from fitting into the cup in diastole (Fig. 2). In diastole there was a gap $2 \mathrm{~mm}$. wide within and around the entire circumference of the prosthesis at its bearing surface, and the result was acute insufficiency. After searching the literature we have not found a similar case. As the result of a careful analysis and reconstruction of the technique we used during the operation we are convinced that bridg- 


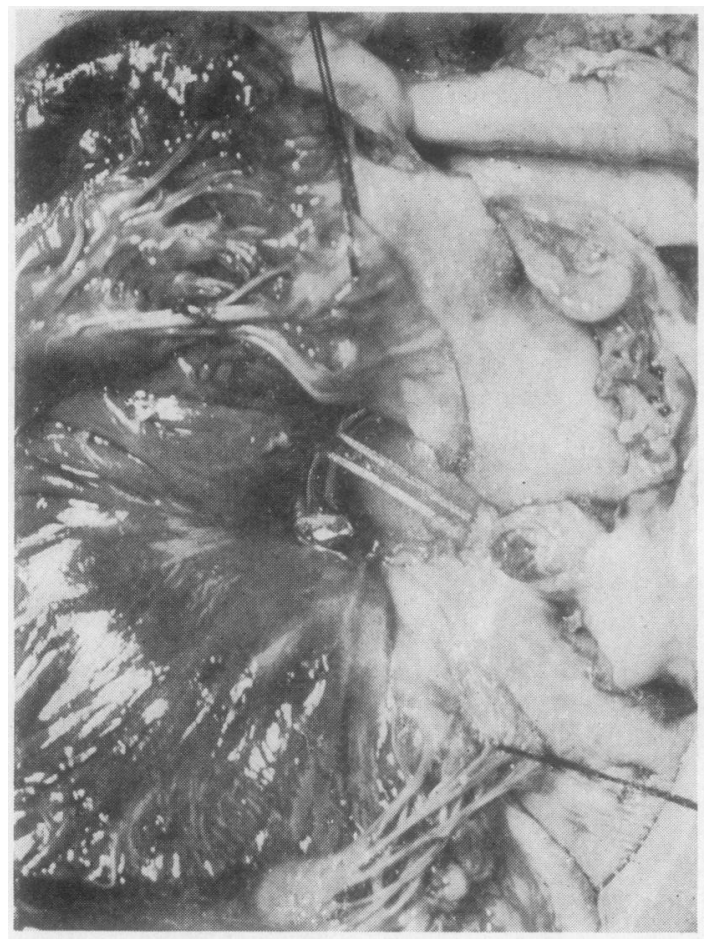

FIG. 2. Post-mortem findings. View of the implanted prosthesis from the left ventricle. Stretched across the base of the prosthesis is the stitch fibre which prevented the ball from fitting into the prothesis saddle in diastole. ing of the base of the prosthesis by the stitch can occur at the moment when the prosthesis is inserted into the subcoronary space of the aorta. After the stitches had been passed through the remaining raw edges of the excised cusps and into the teflon cuff of the prosthesis, the latter was inserted into the aorta and the stitches were simultaneously drawn taut in the other direction. The prosthesis was then lowered into the subcoronary space by tightening the stitches. In our patient one stitch remained loose under the prosthesis and formed a loop, the top of which became fixed across the base of the ball. Thus it happened that the ball was thrown in diastole against the taut stitch instead of against its normal setting. At operation we had noticed nothing unusual. We attributed the acute left ventricular failure to primary insufficiency of the hypertrophic and degenerated heart muscle, and also to the fact that during the operation we had not been able to achieve an efficient coronary artery perfusion.

\section{SUMMARY}

An unusual complication that occurred after implantation of a Starr-Edwards prosthesis is described. One of the stitches that had been inserted to hold the valve in position had been passed across the base of the valve in such a way as to prevent the ball engaging accurately in its seating. 